\title{
Estado del arte sobre la oralidad desde una perspectiva comunica- tiva
}

\section{State of the art on orality from a communicative perspective}

(C) UNAN-Managua

Recibido: 24 de febrero 2021

Aprobado: 24 de marzo 2021

(c) (i) (9)

\section{RESUMEN}

El presente artículo tiene como propósito determinar los avances investigativos en el estudio de la didáctica de la lengua oral desde una perspectiva comunicativa. La metodología utilizada corresponde a las fases de la investigación documental. La primera denominada inicial, se determina el problema (didáctica de la oralidad desde el enfoque comunicativo) y se precisan ocho estudios de tipo tesis y artículo científicos. La segunda fase es la analítica, se establecieron cinco categorías de análisis, destacando los estudios latinoamericanos de didáctica de lengua oral, enfoque comunicativo y el intérvalo temporal del año 2011 a la fecha. La tercera fase es la final, se presentan los principales hallazgos. En relación a la primera categoría, los elementos teóricos comunes de las investigaciones corresponden a oratoria, el lengua verbal y no verbal, y la argumentación. También, sugieren las técnicas mesa redonda, debate y exposiciones para el desarrollo de la oralidad. Asimismo, manifiestan que la dificultad de los docentes radica en la planificación, ejecución y evaluación de la actividad oral. En la segunda categoría, Enfoque Comunicativo, no hay estudios en Nicaragua, los detectados corresponden a propuestas didácticas extranjeras que no fueron aplicadas. Estas hacen énfasis en la capacitación docente sobre la didáctica oral, debido a la centralización por la escritura y lectura. Por último, se presentan los principales vicios detectados en la revisión documental, destacan el escaso estudio de las distintas modalidades discursivas, la falta de inserción de las TIC en la didáctica oral, la nula adopción de un modelo o enfoque determinado, la carente vinculación del Enfoque Comunicativo con el Modelo por Competencias y la falta de propuestas didácticas para el desarrollo de las habilidades comunicativas a niños con necesidades especiales.

Palabras claves: didáctica de la oralidad, enfoque, comunicación
Víctor Alfonso Loáisiga Manzanarez Investigador independiente alfonsoloaisiga@gmail.com

https://orcid.org/0000-0001-6493-7319

\section{SUMMARY}

The purpose of this article is to determine the research advances in the study of the didactics of oral language from a communicative perspective. The methodology used corresponds to the phases of documentary research. The first, called initial, determines the problem (didactics of orality from the communicative approach) and eight scientific thesis and article type studies are required. The second phase is the analytical one, five categories of analysis were established, highlighting the Latin American studies of oral language didactics, communicative approach and the time interval from 2011 to date. The third phase is the final one, the main findings are presented. In relation to the first category, the common theoretical elements of the investigations correspond to oratory, verbal and nonverbal language, and argumentation. Also, they suggest the round table techniques, debate and expositions for the development of orality. Likewise, they state that the teachers' difficulty lies in the planning, execution and evaluation of the oral activity. In the second category, Communicative Focus, there are no studies in Nicaragua, those detected correspond to foreign didactic proposals that were not applied. These place emphasis on teacher training on oral didactics, due to the centralization of writing and reading. Finally, the main vices detected in the documentary review are presented, highlighting the scarce study of the different discursive modalities, the lack of insertion of ICT in oral didactics, the null adoption of a specific model or approach, the lack of linkage of the Communicative Approach with the Competency Model and the lack of didactic proposals for the development of communication skills in children with special needs.

Keywords: didactics of orality, approach, communication 


\section{INTRODUCCIÓN}

Este estudio es una investigación de tipo documental, se estructura a partir de la propuesta de Cifuentes, Osorio y Morales (1993) (como cita Guevara, 2016) al referir tres fases de recopilación y análisis de información. La primera fase, es denominada inicial, implicó la identificación del área problemática, en este caso, didáctica de la lengua oral desde una perspectiva comunicativa, y la recopilación de la información en diferentes fuentes académica, se obtuvo ocho estudios, los cuales siete corresponden a tesis de investigación, tres a nivel de licenciatura, dos a maestría, una en doctorado y un artículo científico.

La segunda fase, es denominada analítica, en esta se establecen las categorías de análisis de los documentos. En lo que respecta a este estudio, se formularon cinco criterios, el primero fue didáctica de la lengua oral, el segundo estudio latinoamericanos, el tercero enfoque comunicativo, el cuarto estudios en lengua materna y el quinto un intérvalo temporal de investigaciones comprendido del año 2011 a la fecha.

Es importante destacar lo planteado por Morales (1993) al mencionar que en esta fase debe de haber una reconstrucción teórica a partir de las relaciones de los argumentos descriptivos en categorías, subcategorías y tendencias (como cita Guevara, 2016). Por esta razón, en este estado del arte se establecieron las subcategorías temáticas: didáctica de la expresión oral, enfoque comunicativo y estudios en lengua materna. Además, la ubicación geográfica (nacionales o internaciones) de las investigaciones.

La tercera fase es dominada final, en esta según Hoyos (2000) se precisa la interpretación de los categorías y subcategorías temáticas establecidas. Asimismo, debe de proporcionar datos investigativos nuevos a través del establecimiento de hipótesis, vacíos, limitaciones, tendencias y logros (como cita Guevara, 2016). En lo que respecta a este proceso, los resultados se presentaron a partir del contraste y comparación de las categorías de análisis establecida en la fase dos desde un enfoque teórico y metodológico, esto permitió establecer los criterios propuestos por Hoyos. Además, se estructuraron gráficos y tablas para determinar categorías comunes desde una perspectiva geográfica y temporal.

\section{DESARROLLO}

\section{Principales conceptos y teorías}

\subsection{Enfoque comunicativo}

Larsen-Freeman (1993) manifiestan que ofrecer una definición exacta del Enfoque Comunicativo es muy difícil, sin embargo, manifiestan que hablar es detenerse a mirar y escuchar la realidad y reflexionar sobre esta, es decir, la enseñanza de la comunicación eficiente (como cita Brenes, 2011). De este planteamiento se 
infieren dos situaciones, la primera, que el Enfoque Comunicativo tiene como objeto de estudio el uso de la lengua en un contexto y la segunda, las reflexiones de este uso considerando elementos extralingüísticos.

Es relevante citar las palabras de Espinoza y Cortez 2019, al explicar:

El Enfoque Comunicativo es una propuesta didáctica para la enseñanza de las lenguas y de la literatura, que subraya como objetivo esencial de esta educación lingüística y literaria la mejora de dicha competencia comunicativa de los alumnos, es decir, de su capacidad para comprender y producir enunciados adecuados con intenciones diversas de comunicación en contextos comunicativos heterogéneos (p.13).

El planteamiento anterior, confirma que el enfoque en mención es una propuesta didáctica utilizada para las lenguas, haciendo referencia no solo a la L2, sino, al desarrollo de las competencias comunicativas de la lengua materna.

Los principios que rigen el Enfoque Comunicativo son propuestos por Romeu, 2006), este determina cinco. El primero refiere que el lenguaje es el medio esencial de comunicación humana, desarrollo personológico y sociocultural. El segundo, es la relación entre el discurso, la cognición y la sociedad. El tercero, el carácter contextualizado del estudio del lenguaje. El cuarto, el estudio del lenguaje como práctica de un grupo o estrato social. El quinto, el carácter interdisciplinario, multidisciplinario, y transdisciplinario y a su vez autónomo sobre el estudio del lenguaje (como cita Espinoza y Cortez, 2019). En síntesis, todos los principios se fundamentan en torno al lenguaje y su uso en diversos contextos considerando su análisis desde diferentes perspectivas del lenguaje.

El Enfoque Comunicativo tiene de manera general seis finalidades según lo expuesto por (Barrera 2014). La primera finalidad es la necesidad de manejar cada principio que lo rige, así como el sistema de categorización con el que se maneja, como por ejemplo los distintos tipos de significado y sentido, texto, discurso, contextos de elocuencia y las distintas tipologías textuales. La segunda es la necesidad de contribuir con estrategias que ayuden a desarrollar varias habilidades de comprensión análisis y elaboración de textos aplicados en casos diferentes.

La tercera finalidad es la necesidad de dar explicación en base al reconocimiento de las propiedades de la textualidad, la progresión temática, pertinencia, coherencia, intertextualidad y ciertos elementos que estudiándolos de manera individual no resultaría eficiente. La cuarta finalidad, es la necesidad de brindar pautas de estudio con el fin de integrar los distintos actos semánticos y pragmáticos con los gramaticales. La última finalidad es interpretar las distintas investigaciones lingüísticas de manera interdisciplinaria, transdisciplinaria y multidisciplinaria, en base a análisis integradores que no lleguen a ser interpretadas como reduccionismo de 
concepciones lingüísticas tradicionales (como cita Espinoza y Cortez 2019). Evidentemente, según lo expuesto por el autor, el Enfoque Comunicativo se rige por la funcionalidad de los estudiantes a partir de la adquisición de una determinada competencia lingüística.

\subsection{Competencia Comunicativa}

El acto comunicativo es un proceso de interacción entre interlocutores en un contexto determinado, todos nos comunicamos diariamente con diferentes propósitos, contextos e intenciones, es ahí donde la competencia comunicativa se vuelve relevante para alcanzar este fin. Hymes (1972, como cita Brenes 2011,) ratifica esta premisa al plantear:

La competencia comunicativa es lo que el hablante necesita conocer para ser comunicativamente competente en una comunidad hablante, de aquí que debe plantearse desde los tres aspectos fundamentales de la comunicación: expresión lingüística, expresión vocal y expresión corporal y la interrelación de ellos para lograr asertividad comunicativa en contextos de comunicación diversos, de aquí que sea considerada como una de las capacidades capitales; lo que es común a la competencia son los conocimientos (conocer), habilidades y aptitudes(hacer), actitudes (ser) y las normas y valores (convivir) (p.114).

De la cita anterior, se puede deducir que la competencia comunicativa no solo se rige por el dominio estructural de la lengua, sino por la reflexión de los códigos en su uso considerando elementos contextuales, actitudinales y procedimentales.

La competencia comunicativa está estructurad a partir de seis componentes, todos interrelacionados para lograr un mayor aprovechamiento en el uso de la lengua de parte de los estudiantes. De acuerdo con Canales y Swiain (1980) el primer componente es el lingüístico o gramatical, se encarga del estudio del código lingüístico. El segundo, es el sociolingüístico, refiere al conocimiento de las variables socioculturales que condicionan los intercambios comunicativos. El tercero, el componente discursivo, alude a la habilidad para expresar de manera coherente la información considerando la gestión temática, la reciprocidad comunicativa, la organización del mensaje y el objetivo comunicativo (como cita Brenes, 2011).

Asimismo, en la opinión de los mismos autores, el cuarto componente es estratégico, se estructura a partir del conocimiento y habilidad para evitar las dificultades comunicativas. En lo que respecta a la quinta competencia, es la literaria, refiere los conocimientos, habilidades y hábitos que hacen posible el uso y disfrute de los textos literarios. Por último, se menciona el componente semiológico, que Incluye conocimientos, habilidades y actitudes que favorecen una interpretación crítica de los usos y formas de los medios de comunicación de masas y de la publicidad (como cita Brenes, 2011). 


\subsection{La expresión oral}

Es fundamental para iniciar este acápite mencionando el planteamiento Oshanam (1996), este sostiene que en las aulas de clase se enfatiza en la idea de que solo el análisis gramatical garantiza un desarrollo armónico del habla, se está frente una teoría que el teórico denomina Teoría Gramatical. Asimismo, el autor sintetiza las principales practicas por los docentes en la enseñanza-aprendizaje de la lengua oral, primeramente, tenemos la practicas basadas en estimular la narración a práctica basada en estimular la narración que presenta dos formas de actuación, narración con presentación o ausencia de modelo; y presencia o ausencia de refuerzo justificado.

Por otro lado, Castillo (2008) argumenta que los principales problemas presentes en los estudiantes en relación a la expresión oral, manifestado por las últimas investigaciones en Latinoamericana y España, se resumen: vocabulario poco enriquecido, muletillas, vicios lingüísticos como dequeísmo y queísmo, fenómenos fonéticos de supresión o de incrementación frecuentes, preferencia de un registro lingüístico informal coloquial, desarrollo incompleto del tema que se discute, dicción imprecisa, excesiva gesticulación, tono de voz bajo o muy alto, mezcla de ideas, escasa atención, falta de comprensión en lo que se les dice, timidez a la hora de hablar frente a los demás, falta de fluidez verbal, ideas incoherentes y desorganizadas y ausencia de estrategias para organizar el discurso.

\subsection{Trabajos realizados en el área}

El proceso de investigación documental se estructuró a partir de las categorías de análisis mencionada en la metodología, de estos, subyacen las subcategorías temáticas: didáctica de la expresión oral, enfoque comunicativo y estudios en lengua materna, a manera de síntesis la siguiente figura resume lo planteado:

Figura 1

Clasificación de las fuentes según categoría temática

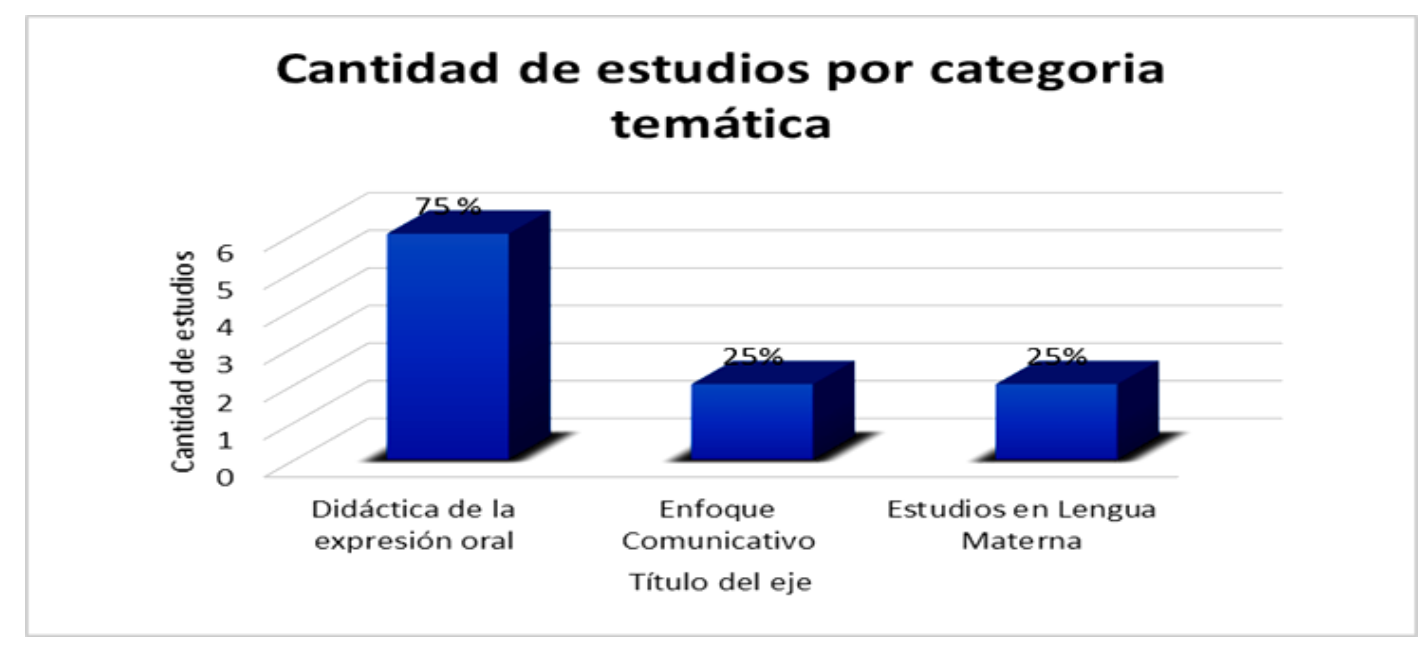


De la figura anterior, se puede inferir las siguientes premisas. En primer lugar, de los ocho estudios analizados, el 75\% equivalen a la categoría didáctica de la expresión oral desarrolladas en los países de Colombia, Chile, Ecuador, Nicaragua, Costa Rica y Perú. En segundo lugar, la categoría Enfoque Comunicativo aplicado a la enseñanza de la lengua materna corresponde a un 25\% en la revisión bibliográfica, esto indica que su aplicación ha sido limitada para el desarrollo de las habilidades del lenguaje desde las aulas de clase.

Asimismo, es fundamental destacar, que la mayoría de estudios, corresponden a la enseñanza de Lenguas Extranjeras (L2), solo se constató su aplicación en lengua materna en la propuesta de Brenes (2011) en su tesis doctoral desarrollada en el año 2009 en Cartago, Costa Rica. Esta se planteó como objetivo el desarrollo de la expresión oral y comprensión auditiva de los estudiantes de educación diversificada a partir del Enfoque Comunicativo. También, Cortez (2019) realiza su tesis de licenciatura para el desarrollo de las habilidades del lenguaje a partir del Enfoque Comunicativo.

En tercer lugar, en el país de Nicaragua, se constataron dos estudios para el desarrollo de la expresión oral, el primero propuesto por Blandino y Ruiz (2016) sobre la efectividad de materiales didácticos en la enseñanza de la oralidad en estudiantes de noveno grado del municipio del Tuma, La Dalia. El segundo, fue propuesto por Loáisiga (2020) en su tesis de licenciatura uso de conectores lógicos para el discurso formal de los estudiantes a través del Modelo de Aprendizaje por Esquemas. Es imprescindible destacar, que ninguna de las dos investigaciones consideró aspectos del Enfoque Comunicativo, incluso, la propuesta de Blandino y Ruiz, no precisó ningún modelo a seguir para resolver el problema delimitado.

\section{Principales alcances, metodología y resultados de los estudios revisados según las categorías temáticas}

\subsection{Didáctica de la expresión oral}

El alcance de las investigaciones indica los principales métodos que se aplicaran sobre una realidad problemática para obtener resultados. En lo que refiere a las seis investigaciones analizadas, se corroboró que tres son de tipo exploratorias, la primera es la tesis a nivel de maestría de Zarza (2017) sobre una propuesta didáctica para el desarrollo de la expresión oral, la segunda Casanova y Roldan (2016) en su artículo científico alcances de la enseñanza de la oralidad en la enseñanza media y la tercera un estudio de Melo (2016) sobre estrategias didácticas para el desarrollo de la expresión oral en estudiantes de educación básica.

Ambos estudios exploran las dificultades de la enseñanza de la oralidad en educación media y básica. El primero propone una serie de estrategias a partir de la teoría de la metacognición, el estudio del lenguaje no verbal, la retórica, la argumentación y las habilidades sociales. El segundo, plantea que el lenguaje 
formal debe de prevalecer en el discurso de la enseñanza media, sugiere la técnica de exposición considerando la prosodia y oratoria como elementos esenciales para su planificación. La tercera, presenta una secuencia didáctica basada en los resultados de un diagnóstico aplicado a estudiantes de educación básica. Con relación al alcance descriptivo la propuesta de investigación de Loáisiga (2020) se clasifican en esta categoría. Esta describe un fenómeno determinado (dificultades de expresión oral), hacen una medición de las variables a través de la estructuración de un sistema de categorías según Modelo de Aprendizaje por Esquemas y presenta los resultados a partir de la medición de las mismas (variables) en la intervención didáctica.

La propuesta de investigación a nivel de maestría de García (2017) sobre estrategias activas interdisciplinarias para fortalecer las capacidades comunicativas de la formación de docentes de primaria, es clasificada como explicativa. La aplicación de pretest le permitió conocer las causas de las dificultades en las habilidades comunicativas (hablar, escuchar, escribir y leer), posteriormente la aplicación de estrategias y el análisis de un post test favoreció determinar el avance de los educandos en las habilidades mencionadas. Es fundamental mencionar que la investigación propuesta a nivel de licenciatura por Blandino y Ruiz (2016) sobre la efectividad de los materiales didácticos en la enseñanza de la oralidad en estudiantes de noveno grado del municipio del Tuma, La Dalia, no precisan con el tipo de estudio y enfoque. En relación a la metodología de las investigaciones mencionadas, la tabla 1 se esquematiza todos los elementos de este criterio:

\section{Tabla 1}

Elementos metodológicos de las investigaciones

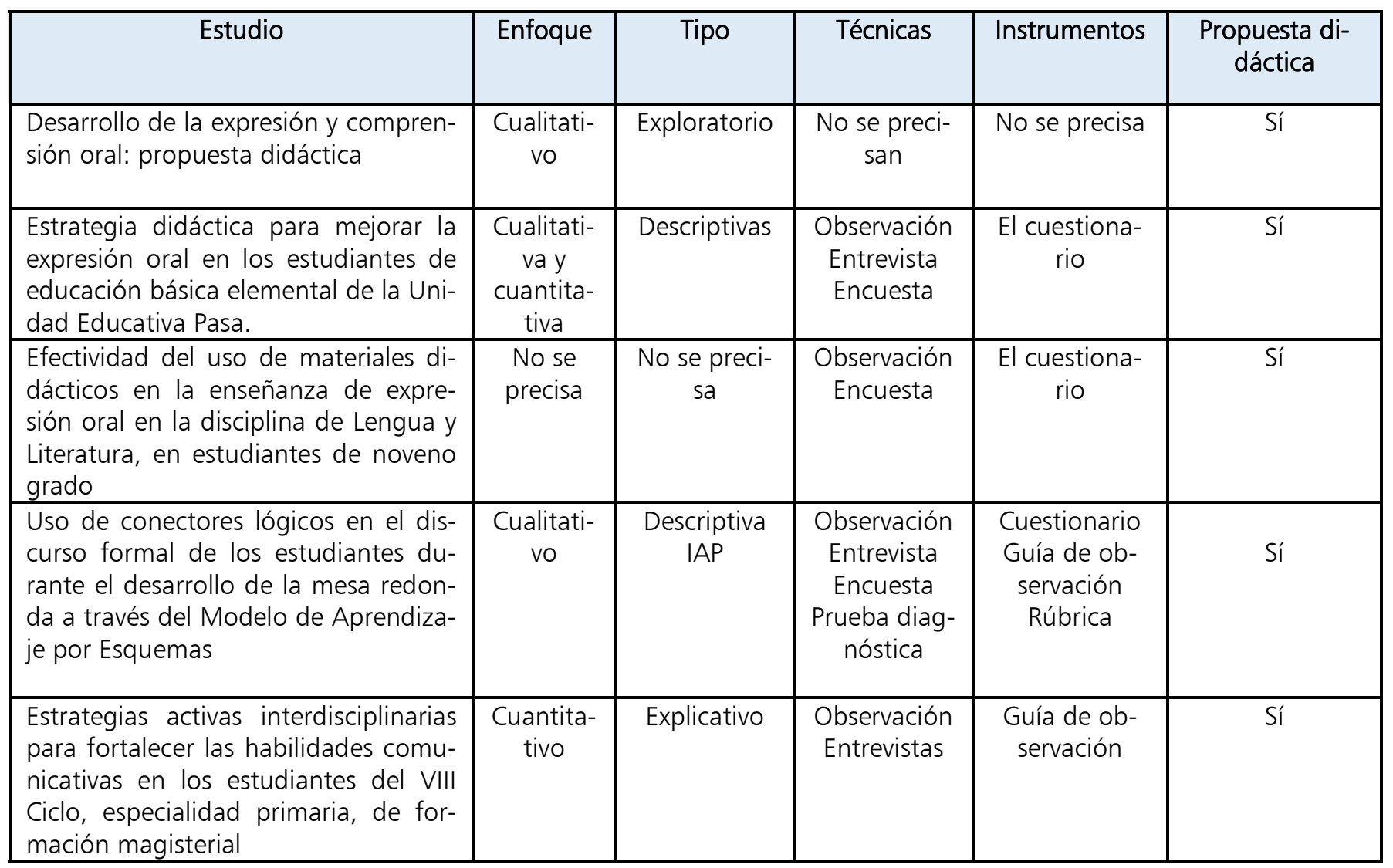


A partir de la tabla anterior, se infiere que el $40 \%$ de las investigaciones analizadas utilizan el enfoque cualitativo, el cuantitativo el $20 \%$ y el mixto (cualitativo y cuantitativo) el 20\%. Además, un 20\% de los estudios no precisa el enfoque de investigación adoptado. Asimismo, la observación, entrevista y encuesta son las técnicas más utilizadas para la recopilación y análisis de la información. En lo que respecta a los instrumentos utilizados, el cuestionario, la guía de observación y la rúbrica fueron los comunes entre todos los estudios.

Lo anteriormente planteado, evidencia factores comunes entre todas las investigaciones. El primero, todas tratan de incidir en el objeto de estudio (dificultades en la oralidad) desde diferentes perspectivas. En segundo, hay propuestas de intervención didáctica para desarrollar la habilidad lingüística de la expresión oral, la mayoría se fundamentan en el estudio de los elementos verbales y paraverbales de la oralidad, los actos del habla, la teoría de la argumentación en el discurso, la comprensión de las situaciones comunicativas en contextos diversos, y la oratoria. Además, autores como Zarza (2017) proponen la técnica debate para la reflexión metacognitiva del discurso oral a través del modelado. También, Casanova y Roldán (2016) sugieren la exposición como mecanismo para la producción de los géneros discursivos. Por último, Loáisiga (2020) manifiesta que la mesa redonda es una de las técnicas más asequibles para el desarrollo del discurso formal.

Los resultados de las investigaciones analizadas se presentan a partir de dos proposiciones. La primera, relacionada a los objetivos de las intervenciones didácticas propuestas y la segunda desde la observación de la práctica docente. En lo que respecta al primer criterio, la mayoría de investigadores concluyen que el planteamiento de propuestas metodológicas debe de estar enfocado en situaciones concretas de comunicación, se debe de analizar el contexto, los propósitos sociales, el modo de organización y los recursos lingüísticos.

También, el éxito del desarrollo de la expresión oral consiste en integrar elementos verbales y paraverbales de la oralidad en las estrategias didácticas, esto permite que los estudiantes desarrollen el pensamiento crítico (metareflexión) del código lingüístico. Además, se determinó que el discurso que más prevalece en los estudiantes de educación media es el informal, no hay dominio de argumentos, ni los mecanismos para organizar y comprender discursos formales. Por último, se deben de aplicar técnicas como la mesa redonda, debates, exposiciones y conversatorios para estimular los géneros discursivos en los discentes.

En relación a la práctica docente a partir de las observaciones de los investigadores se concluyó que la mayoría tiene un desconociendo de la planificación, ejecución y evaluación de las actividades orales en el aula de clase. Además, solo utilizan como recursos didácticos los libros de textos, diccionarios, pizarra y marcadores. En síntesis, la mayor atención de ellos está en el fortalecimiento de las habilidades de escritura y lectura.

\subsection{Enfoque Comunicativo}

En lo que respecta a esta categoría se presenta el alcance de dos investigaciones, la primera propuesta por Brenes (2011) en su tesis doctoral. La segunda, a nivel de licenciatura presentada por Espinoza y Cortez (2019). El alcance de la tesis de Brenes es de tipo no experimental, debido a que fundamenta en la observación de situaciones ya existentes en función de las variables de análisis establecidas, en este caso las activida- 
des didácticas, los recursos didácticos, las técnicas evaluativas y la capacitación docente para el desarrollo de la expresión oral y la comprensión auditiva en educación diversificada a la luz del enfoque comunicativo.

El estudio de Espinoza y Cortez (2019) es de tipo exploratoria, ya que se encargó de examinar un problema de investigación para establecer prioridades de estudio a futuro, en este caso, se evaluó el desempeño docente en el área de Lengua y Literatura a partir de la aplicación del Enfoque Comunicativo. Además, se planteó una propuesta para resolver hasta cierto grado las dificultades detectadas en el proceso de planificación, ejecución y evaluación de las competencias lingüísticas (leer, escribir, hablar y escuchar).

En relación a la metodología de las investigaciones mencionadas, el siguiente cuadro esquematiza todos los elementos de este criterio:

\section{Tabla 2}

Elementos metodológicos de las investigaciones según Enfoque Comunicativo

\begin{tabular}{|c|c|c|c|c|c|}
\hline \multirow[t]{2}{*}{ Nombre del estudio } & \multicolumn{5}{|c|}{ Elementos metodológicos } \\
\hline & Enfoque & Tipo & Técnicas & Instrumentos & $\begin{array}{l}\text { Propuesta } \\
\text { didáctica }\end{array}$ \\
\hline $\begin{array}{c}\text { Desarrollo de la expresión oral } \\
\text { y la comprensión auditiva co- } \\
\text { mo parte de las competencias } \\
\text { comunicativas y desde el en- } \\
\text { foque comunicativo, en estu- } \\
\text { diantes de educación diversifi- } \\
\text { cada de colegios públicos de } \\
\text { Cartago en el } 2009\end{array}$ & Cuantitativo & No experimental & $\begin{array}{l}\text { Encuesta } \\
\text { autoadmi- } \\
\text { nistrada } \\
\text { Observa- } \\
\text { ción } \\
\text { Entrevista }\end{array}$ & $\begin{array}{l}\text { Cuestionarios } \\
\text { Hojas de obser- } \\
\text { vación en el } \\
\text { aula }\end{array}$ & Sí \\
\hline $\begin{array}{c}\text { Estrategia didáctica para me- } \\
\text { jorar la expresión oral en los } \\
\text { estudiantes de educación bá- } \\
\text { sica elemental de la Unidad } \\
\text { Educativa Pasa. }\end{array}$ & Cualitativo & Exploratorio & $\begin{array}{l}\text { Observa- } \\
\text { ción } \\
\text { Diario de } \\
\text { campo } \\
\text { Prueba } \\
\text { diagnóstica } \\
\text { Análisis } \\
\text { documental } \\
\text { Grupo focal }\end{array}$ & $\begin{array}{c}\text { El cuestionario } \\
\text { Lista de cotejo } \\
\text { Guía de obser- } \\
\text { vación }\end{array}$ & Sí \\
\hline
\end{tabular}

De la tabla anterior, se infieren los siguientes cuestionamientos. En primer, que se puede estudiar y aplicar el enfoque comunicativo desde un enfoque cualitativo y cuantitativo según los objetivos de cada investigador. Evidentemente, Brenes (2011) toma como objeto de estudio la práctica docente y la percepción sobre la clase de lengua del municipio de Cartago, Costa Rica. Mientras, que Espinoza y Cortez (2019) solo evalúan el desarrollo de las habilidades de la lengua en una sección de clase determinada aplicando el enfoque en estudio. En segundo lugar, los instrumentos más utilizados para trabajar este campo fue la observación, encuesta y entrevista.

Los resultados de las dos investigaciones analizadas se presentan a partir de los criterios relacionados a las intervenciones didácticas y la práctica docente. En relación al primer criterio, es relevante resaltar que ambos estudios solo presentan propuestas de intervención didáctica según las dificultades detectadas en la diagnosis de la 
muestra precisada, no hay aplicación. La investigación de Brenes manifiesta en su propuesta elementos para las variables actividades didácticas, recursos didácticos y técnicas evaluativas para el desarrollo de la expresión oral y comprensión auditiva desde el Enfoque Comunicativo.

En relación a la primera variable menciona que las actividades didácticas deben de estructurase a partir de las adaptaciones de las necesidades comunicativas de los estudiantes, estudio del registro lingüístico, paralingüísticos y adaptación al grado de madurez. Además, sugiere las técnicas de dramatización, narración oral, el juego de roles, la entrevista, el diálogo, el foro, estudios de casos y la excursión. Asimismo, desde las sugerencias de los recursos utilizados se deben de crear situaciones dialogales y monologales. Por último, como técnicas evaluativas sugiere el portafolio, la observación, la implementación de los diferentes tipos de evaluación.

Por su parte, Espinoza y Cortez (2019) se limitan a ofrecer una solución a nivel institucional para mejorar la asignatura de Lengua y Literatura desde Enfoque Comunicativo. Su propuesta consiste en la realización de un proceso de capacitación dirigidos a los directivos y personal docente sobre el enfoque en análisis, este se divide en cuatro fases: diagnóstico, taller del experto, planificación docente, Lesson Study y evaluación.

\subsection{Vacíos cognitivos identificados}

El análisis documental de los ochos estudios propuestos permitió identificar algunos vacíos cognitivos presentes en la mayoría de investigaciones. El primero está relacionado a la modalidad discursiva desarrollada desde las propuestas didácticas, la mayoría de autores, se enfocan en el discurso argumentativo, adaptado desde las técnicas orales de la mesa redonda, debates, exposiciones etc., obvian las demás modalidades discursivas como la exposición, narración y descripción.

El segundo se hace referencia al uso de las TIC para el desarrollo de las actividades orales, en el análisis documental solo se tiene constancia del estudio de Zarza (2017) y Loáisiga (2020) al utilizar videos para práctica del modelado de situaciones comunicativas, no hay ninguna alusión a una aplicación móvil en las propuestas didácticas planteadas por los especialistas.

El tercer vacío encontrado es la ausencia de modelos de enseñanza para el desarrollo de la oralidad en los diferentes sistemas educativos, solo se verificó la propuesta de Brenes (2011), Espinoza y Cortez (2019) desde el Enfoque Comunicativo. También, Loáisiga (2020) desde el Modelo de Aprendizaje por Esquemas. Los demás estudios, solo delimitaron su problema, analizaron sus variables y aplicaron sus líneas de intervención didáctica sin apagarse a un enfoque o modelo preciso.

En relación al Enfoque Comunicativo, se detectaron los siguientes vacíos. En primera instancia, las únicas dos propuestas en lengua materna determinadas en este estudio fueron las de Brenes, Espinoza y Cortez, estas no se aplicaron a la realidad problemática definida, solo fueron sugerencias para enfrentar los hechos delimitados en los instrumentos de investigación de la fase diagnóstica. También, dicho enfoque 
está orientado a la expresión oral y auditiva, no hay estudios en las demás habilidades del lenguaje (lectura y escritura), Espinoza y Cortez, solo brindan algunas pautas para poderlas integrar.

Asimismo, el objetivo del Enfoque Comunicativo es desarrollar la competencia comunicativa, sin embargo, no se constataron estudios que vincularan todas las habilidades lenguaje en una sola propuesta didáctica. Además, relacionarlas con otros tipos de competencia desde el Enfoque por Competencias aplicados en países como Nicaragua. Otro aspecto importante, es que ninguno de los estudios revisados se orienta el desarrollo de la competencia oral para estudiantes con necesidades especiales en los diversos ambientes educativos.

Es interesante mencionar, desde el punto de vista del alcance de investigación, la mayoría de estudios son de tipo exploratorio, hay un mínimo con el enfoque descriptivo y explicativo, y ninguno con el enfoque correlacional. Esto indica, que se deben de realizar propuestas didácticas a partir de los alcances no estudiados, lo que permitirá tener una mayor explicación de los fenómenos (problema de investigación) para probar hipótesis y establecer de manera objetivas nuevas líneas de investigación.

\section{CONCLUSIONES}

El paradigma de aprendizaje oral de la lengua por naturalidad es la mayor barrera para los docentes. En su mayoría, no consideran necesaria el estímulo de esta competencia comunicativa en los estudiantes. Evidentemente, hay una supremacía de potenciar las habilidades de lectura y escritura de diversos tipos de textos, sin considerar la interrelación de las competencias lingüísticas en el desarrollo de la comunicación.

Estas circunstancias afectan de manera negativa en los estudiantes, iniciando desde la comprensión hasta la estructuración de discursos según las necesidades comunicativas actuales. Además, hay barreras en la adquisición y retroalimentación del conocimiento (proceso de fijación), limitantes en las relaciones sociales por la incomprensión de los elementos de la comunicación, el registro y la organización del discurso, obstáculos en la resolución de conflictos y exteriorización de emocionales debido al escaso estímulo de la escucha activa.

En Nicaragua, se constató a través de la revisión documental la carencia de investigaciones en esta área. Además, las pocas existentes no precisan un modelo de aprendizaje para el desarrollo de la oralidad, no hay incursión de las TIC en las actividades orales y no existe ninguna investigación referida a la competencia comunicativa en niños con necesidades especiales.

Por tanto, es necesario crear una propuesta didáctica dirigida a la capacitación docente de secundaria a partir de la metodología del Enfoque Comunicativo para el desarrollo de la competencia oral integrando las TIC. Además, es primordial analizar las variaciones de esta propuesta según la identificación de estudiantes con necesidades especiales en las aulas de clase. El trabajo de campo se debe de constituir a partir del análisis de la práctica docente y el establecimiento de alternativas didácticas para analizarse en la aplicación a los discentes según las competencias adquiridas por el claustro. 


\section{REFERENCIAS}

Blandino-Pérez, L y Ruiz-Duarte, T. (2017). Efectividad del uso de materiales didácticos en la enseñanza de expresión oral en la disciplina de Lengua y Literatura, en estudiantes de noveno grado " $B$ ", turno vespertino, del centro escolar 14 de Septiembre, Municipio El Tuma-La Dalia Departamento de Matagalpa, segundo semestre, del año lectivo 2016. [ Tesis de licenciatura, Universidad Nacional Autónoma de Nicaragua, FAREM-Matagalpa] Archivo digital https://repositorio.unan.edu.ni/5037/

Brenes, R. (2011). Desarrollo de la expresión oral y la comprensión auditiva como parte de las competencias comunicativas y desde el enfoque comunicativo, en estudiantes de educación diversificada de colegios públicos de Cartago en el 2009 [ Tesis de doctorado, Universidad Nacional Estatal a Distancia] Archivo digital https://repositorio.uned.ac.cr/reuned/handle/120809/1004

Castillo, J. (2008). El desarrollo de la expresión oral a través del taller como estrategia didáctica globalizadora. Sapiens. Revista Universitaria de Investigación, 9(1), 179-203. file:///E:/Downloads/ DialnetEIDesarrolloDeLaExpresionOralATravesDelTallerComoE-2781929.pdf

Dijk, V. (1981). El análisis del discurso en la organización y representación de la información. Conocimiento de los elementos teóricos. Revista Acimed; 14 (3), 68-97.

Espinoza, K y Cortez, V. (2019). Estrategia metodológica para el desarrollo del proceso de enseñanza aprendizaje de la lengua y literatura desde un enfoque comunicativo [ Tesis de licenciatura, Universidad Nacional de Educación] Archivo digital. http://repositorio.unae.edu.ec/bitstream/56000/1118/1/El\% 20enfoque\%20comunicativo.pdf

Guerrero-García, J. (2014). Estrategias activas interdisciplinarias para fortalecer las habilidades comunicativas en los estudiantes del VII Ciclo, especialidad primaria, de formación magisterial del JESSP Rafael Hoyos Rubio de la Provincia de San Ignacio, 2014 [ Tesis de maestría, Universidad Nacional de Cajamarca] Archivo digitalhttps://repositorio.unc.edu. pe/bitstream/handle/UNC/1332/ESTRATEGIAS\%20ACTIVAS\% 20INTERDISCIPLINARIAS\%20PARA\%20FORTALECER\%20LAS\%20HABILIDADES\%20COMUNICATIVAS\% 20EN\%20LOS\%20ESTU.pdf? sequence $=1$ \&isAllowed $=y$

Guevara, P. (2016). El estado del arte en la investigación: Lanálisis de los conocimientos acumulados o indagación por nuevos sentidos?, Revista Folios. (44), 165-179. http://www.scielo.org.co/pdf/folios/n44/ n44a11.pdf

Loáisiga, V. (2020). Uso de conectores lógicos en el discurso formal de los estudiantes durante el desarrollo de la mesa redonda a través del Modelo de Aprendizaje por Esquemas [ Tesis de licenciatura, Universidad Nacional Autónoma de Nicaragua, Managua] Archivo digital https://repositorio.unan.edu.ni/13651/

Lomas, C., Osorio, A., y Tusón, A. (1992). Ciencias del lenguaje: competencia comunicativa y enseñanza de la lengua, Revista Signos, 7, 27-53. http://www.quadernsdigitals. net/index.php? accionMenu=hemeroteca.VisualizaArticulolU.visualiza\&articulo_id $=590$ 
Melo-Villagomes, G. (2016). Estrategias Didácticas para mejorar la expresión oral en estudiantes de educación básica elemental de la unidad educativa Pasa [ Tesis de licenciatura, Pontificia Universidad Católica del Ecuador] Archivo digital https://repositorio.pucesa.edu.ec/bitstream/123456789/1753/1/76252.pdf

O'shanahan, I. (1996). Enseñanza del lenguaje oral y las teorías implícitas del profesorado. Soportes Audiovisuales e informáticos [ Tesis doctoral, Universidad de la Laguna] Archivo digital ftp://tesis.bbtk.ull.es/ ccssyhum/cs27.pdf

Real Academia Española (2014). Diccionario de la lengua española. 23. a edición. Madrid: Espasa Libros.

Zarza, D. (2017). El desarrollo de la expresión y comprensión oral: propuesta didáctica [ Tesis de maestría, Universidad Internacional de la Rioja] Archivo digital https://reunir.unir.net/bitstream/ handle/123456789/5109/ZARZA\%20GOMEZ\%2C\%20DAGOBERTO.pdf?sequence=1 \&isAllowed=y

Zebadúa, M y García, E. (2012). Cómo enseñar a hablar y escuchar en el salón de clase. Editorial UNAM. https://portalacademico.cch.unam.mx/materiales/libros/pdfs/librocch_hablarescuchar.pdf 